\title{
Dynamics of water in prussian blue analogues: Neutron scattering study
}

\author{
V. K. Sharma, ${ }^{1}$ S. Mitra, ${ }^{1}$ N. Thakur, ${ }^{1}$ S. M. Yusuf, ${ }^{1}$ Fanni Juranyi, ${ }^{2}$ \\ and R. Mukhopadhyay ${ }^{1, a)}$ \\ ${ }^{1}$ Solid State Physics Division, Bhabha Atomic Research Centre, Mumbai 400085, India \\ ${ }^{2}$ Laboratory for Neutron Scattering, Paul Scherrer Institut, Villigen, Switzerland
}

(Received 2 June 2014; accepted 9 July 2014; published online 21 July 2014)

\begin{abstract}
Dynamics of crystal water in Prussian blue $(\mathrm{PB}), \mathrm{Fe}(\mathrm{III})_{4}\left[\mathrm{Fe}(\mathrm{II})(\mathrm{CN})_{6}\right]_{3} \cdot 14 \mathrm{H}_{2} \mathrm{O}$ and its analogue Prussian green $(\mathrm{PG})$, ferriferricynaide, $\mathrm{Fe}(\mathrm{III})_{4}\left[\mathrm{Fe}(\mathrm{III})(\mathrm{CN})_{6}\right]_{4} \cdot 16 \mathrm{H}_{2} \mathrm{O}$ have been investigated using Quasielastic Neutron Scattering (QENS) technique. PB and its analogue compounds are important materials for their various interesting multifunctional properties. It is known that crystal water plays a crucial role towards the multifunctional properties of Prussian blue analogue compounds. Three structurally distinguishable water molecules: (i) coordinated water molecules at empty nitrogen sites, (ii) non-coordinated water molecules in the spherical cavities, and (iii) at interstitial sites exist in $\mathrm{PB}$. Here spherical cavities are created due to the vacant sites of $\mathrm{Fe}(\mathrm{CN})_{6}$ units. However, $\mathrm{PG}$ does not have any such vacant $\mathrm{N}$ or $\mathrm{Fe}(\mathrm{CN})_{6}$ units, and only one kind of water molecules, exists only at interstitial sites. QENS experiments have been carried out on both the compounds in the temperature range of $260-360 \mathrm{~K}$ to elucidate the dynamical behavior of different kinds of water molecules. Dynamics is found to be much more pronounced in case of PB, compared to PG. A detailed data analysis showed that localized translational diffusion model could describe the observed data for both PB and PG systems. The average diffusion coefficient is found to be much larger in the PB than PG. The obtained domain of dynamics is found to be consistent with the geometry of the structure of the two systems. Combining the data of the two systems, a quantitative estimate of the dynamics, corresponding to the water molecules at different locations is made. (C) 2014 AIP Publishing LLC. [http://dx.doi.org/10.1063/1.4890722]
\end{abstract}

\section{INTRODUCTION}

Molecular magnets have many useful additional properties over conventional magnets such as low density, flexible, magneto-optical properties, and biocompatibility., ${ }^{1,2}$ They also offer the opportunity of being able to tune the transition temperature at which the material becomes ferromagnetic by fine tuning the chemical structure. Recently, there has been intense research in the field of molecular magnets based on hexacyanometalates. ${ }^{3-8}$ Hexacyanometallates can be represented by the general formula $A_{\mathrm{x}}\left[B(\mathrm{CN})_{6}\right]_{\mathrm{y}} \cdot \mathrm{zH}_{2} \mathrm{O}$, where $A$ and $B$ are $3 \mathrm{~d}$ transition metal ions. Hexacyanometallates possess $f c c$ structure in which $A$ and $B$ are surrounded octahedrally by $N$ and $C$ atoms, respectively. When $\mathrm{x} / \mathrm{y}=1$, the first coordinations of $A$ and $B$ are $A[\mathrm{NC}]_{6}$ and $B[\mathrm{CN}]_{6}$, respectively. In this case, water molecules occupy the interstitial positions. ${ }^{6}$ However, when $x / y>1$, some of the $B[\mathrm{CN}]_{6}$ vacancies are vacant and filled by water molecules. In this case, the first coordinations of $A$ and $B$ are $A(\mathrm{NC})_{6-n}$ $(\mathrm{H} 2 \mathrm{O})_{\mathrm{n}}(\mathrm{n}=1-6)$ and $B[\mathrm{CN}]_{6}$, respectively. Three structurally distinguishable water molecules, (i) coordinated water molecules which are coordinated to $A$ octahedra at empty nitrogen $24 \mathrm{e}(x, 0,0)$ site, (ii) non-coordinated water molecules connected by hydrogen bonds to the coordinated ones in the spherical cavity of $\left[\mathrm{B}(\mathrm{CN})_{6}\right]$ at $32 \mathrm{f}(x, x, x)$ site, and (iii) non-coordinated water at $8 \mathrm{c}(1 / 4,1 / 4,1 / 4)$ site of unit cell octants are found in these compounds. ${ }^{7 / 9}$ Prussian blue

\footnotetext{
a) Author to whom correspondence should be addressed. Electronic mail: mukhop@barc.gov.in. Tel.: +91-22-25593754. Fax: +91-22-25515050.
}

analogue (PBA) are important compounds due to their various interesting multifunctional properties upon application of external stimuli, such as temperature, magnetic field, light, pressure, humidity, etc. By varying the humidity, one can tune physical as well as magnetic properties of PBA compounds. ${ }^{5}$ For example, it has been shown that by varying the humidity content, color of cobalt based PBA is found to change between blue and pink, and magnetic interaction is found to switch between ferromagnetic and antiferromagnetic coupling. ${ }^{5}$ Water plays a crucial role towards the multifunctional properties of PBA compounds. In this paper, we focus on the dynamical behavior of water molecules in these technologically important PBA compounds. Earlier we have studied dynamics of water in a Prussian blue analogue, $\mathrm{Cu}_{2} \mathrm{Mn}_{2}\left[\mathrm{Fe}(\mathrm{CN})_{6}\right]_{2.67} \cdot 19 \mathrm{H}_{2} \mathrm{O}$ (Ref. 10) and showed that water molecules existing at different locations show different dynamical behavior. Dynamics of water can be studied via various techniques, such as neutron scattering, ${ }^{11-22}$ PFGNMR, ${ }^{23}$ computer simulation, ${ }^{24}$ etc. Neutron is a powerful probe to study the dynamics in condensed matter as its energy matches with the kinetic energies of atoms in condensed matter. Also systems containing hydrogen atoms are suitable for neutron scattering studies due to their large incoherent scattering cross-section. Earlier we have studied dynamics of water in various media, such as clays, ${ }^{14,15}$ polyamide membranes, ${ }^{16-18}$ porous alumina gel, ${ }^{19}$ etc. using Quasielastic Neutron Scattering (QENS) techniques. In a QENS experiment, information on molecular dynamics is obtained by considering the dynamical structure factor, $\mathbf{S}(\mathbf{Q}, \omega)$, which indicates the probability that an incident 
neutron undergoes a scattering process with an atom by exchanging energy $\hbar \omega$ and momentum $\hbar \mathbf{Q}$. Characteristic times and the spatial extent of the molecular dynamics can be obtained by analyzing the $\omega$ and $\mathbf{Q}$ dependence of $\mathrm{S}(\mathbf{Q}, \omega)$, respectively. The technique of QENS has found a wide use in the study of dynamics of atoms/molecules in condensed matter for its ability to give spatial as well as temporal information on a wide range of time $\left(10^{-10}-10^{-13} \mathrm{~s}\right)$ and length (few angstroms) scales. ${ }^{11}$ This technique provides quantitative as well as qualitative information about the dynamics. The quantitative information entails the information about the correlation time, length scale, and activation energy while qualitative information pertains to the geometrical mechanism of the motion.

Prussian Blue (PB), $\mathrm{Fe}(\mathrm{III})_{4}\left[\mathrm{Fe}(\mathrm{II})(\mathrm{CN})_{6}\right]_{3} \cdot 14 \mathrm{H}_{2} \mathrm{O}$, is the parent compound of the family of hexacyanometallates. Dynamics of water in $\mathrm{PB}, \mathrm{Fe}(\mathrm{III})_{4}\left[\mathrm{Fe}(\mathrm{II})(\mathrm{CN})_{6}\right]_{3} \cdot 14 \mathrm{H}_{2} \mathrm{O}$ as well as its analogue compound, ferriferricynaide, $\mathrm{Fe}$ $(\mathrm{III})_{4}\left[\mathrm{Fe}(\mathrm{III})(\mathrm{CN})_{6}\right]_{4} \cdot 16 \mathrm{H}_{2} \mathrm{O}$ or Prussian green (PG) has been studied using the QENS technique, and discussed here. The structures of these compounds are very well studied using $\mathrm{x}$-ray as well as neutron diffraction techniques. ${ }^{6,9}$ In PB $\left(\mathrm{Fe}(\mathrm{III})_{4}\left[\mathrm{Fe}(\mathrm{II})(\mathrm{CN})_{6}\right]_{3} \cdot 14 \mathrm{H}_{2} \mathrm{O}\right)$, owing to the $4: 3$ (x: y) stoichiometry, the charge neutrality necessitates that $1 / 4$ of the $\mathrm{Fe}(\mathrm{CN})_{6}$ sites are vacant, and they are filled by the water molecules. ${ }^{9}$ As mentioned above, there are three structurally distinguishable water molecules: (i) coordinated water molecules at empty nitrogen sites, (ii) non-coordinated water molecules in the spherical cavities and (iii) at interstitial sites, exist in PB. Out of 14 water molecules, six water molecules are coordinated to $\mathrm{Fe}(\mathrm{III})$ at empty nitrogen sites $24 \mathrm{e}(\mathrm{x}, 0,0)$ site, four non-coordinated water molecules at $32 \mathrm{f}(\mathrm{x}, \mathrm{x}, \mathrm{x})$ site inside the spherical cavity, and the remaining four non-coordinated water molecules are found at $8 \mathrm{c}(1 / 4,1 / 4,1 / 4)$ site of unit cell octants. ${ }^{9}$ Fig. 1(a) shows the typical spherical cavity formed by coordinated water molecules in case of PB. The noncoordinated water molecules are omitted for clarity. The diameter of the cavity, estimated by positions of oxygen atoms of the coordinated water molecules, is $\sim 5.2 \AA$. However, in PG $\left(\mathrm{Fe}_{4}(\mathrm{III})\left[\mathrm{Fe}(\mathrm{III})(\mathrm{CN})_{6}\right]_{4} \cdot 16 \mathrm{H}_{2} \mathrm{O}\right.$ ), owing to the $1: 1$ (x:y) stoichiometry, no $\mathrm{Fe}(\mathrm{CN}) 6$ sites are vacant [Fig. 1(b)]. Ferriferricyanide has only one kind of water molecules, which are non-coordinated and located at the interstitial sites. ${ }^{6}$ The structures of these compounds are very well studied using $\mathrm{x}$-ray as well as neutron diffraction techniques. ${ }^{6,9}$ Here, the dynamics of water in $\mathrm{PG}\left(\mathrm{Fe}(\mathrm{III})_{4}\left[\mathrm{Fe}(\mathrm{III})(\mathrm{CN})_{6}\right]_{4} \cdot 16 \mathrm{H}_{2} \mathrm{O}\right)$ and $\mathrm{PB}\left(\mathrm{Fe}(\mathrm{III})_{4}\left[\mathrm{Fe}(\mathrm{II})(\mathrm{CN})_{6}\right]_{3} \cdot 14 \mathrm{H}_{2} \mathrm{O}\right)$ as studied using the QENS technique is reported. With the specific characteristics of these two samples, we have made quantitative estimates of the dynamics, corresponding to the water molecules at different locations.

\section{EXPERIMENTAL DETAILS}

The polycrystalline samples of $\mathrm{PB}$ and $\mathrm{PG}$ are prepared by the precipitation method. $\mathrm{PB}\left(\mathrm{Fe}(\mathrm{III})_{4}\left[\mathrm{Fe}(\mathrm{II})(\mathrm{CN})_{6}\right]_{3} \cdot 14 \mathrm{H}_{2} \mathrm{O}\right)$ is prepared by mixing $0.1 \mathrm{M}$ aqueous solution of $\mathrm{K}_{4} \mathrm{Fe}(\mathrm{CN})_{6}$, to rapidly stirred aqueous solution of $0.1 \mathrm{M} \mathrm{FeCl}_{3}$. For PG $\left(\mathrm{Fe}_{4}(\mathrm{III})\left[\mathrm{Fe}(\mathrm{III})(\mathrm{CN})_{6}\right]_{4} \cdot 16 \mathrm{H}_{2} \mathrm{O}\right), 0.1 \mathrm{M} \mathrm{K}_{3} \mathrm{Fe}(\mathrm{CN})_{6}$ aqueous solution was slowly added to $0.1 \mathrm{M} \mathrm{FeCl}_{3}$ aqueous solution in the appropriate proportions and the resulting solution was heated up to $325 \mathrm{~K}$. The hot solution was allowed to cool at room temperature and diluted to double of its initial volume after cooling. The obtained precipitates were filtered, washed many times with distilled water, and finally dried at room temperature. These samples are well characterized by $\mathrm{x}$-ray as well as neutron diffraction techniques. The prepared compound is found to be in $f c c$ crystalline phase with $F m 3 m$ space group.

QENS measurements have been carried out on $\mathrm{PB}$ $\left(\mathrm{Fe}(\mathrm{III})_{4}\left[\mathrm{Fe}(\mathrm{II})(\mathrm{CN})_{6}\right]_{3} .14 \mathrm{H}_{2} \mathrm{O}\right)$ and $\quad \mathrm{PG} \quad\left(\mathrm{Fe}_{4}(\mathrm{III})[\mathrm{Fe}(\mathrm{III})\right.$ $\left.(\mathrm{CN})_{6}\right]_{4} \cdot 16 \mathrm{H}_{2} \mathrm{O}$ ) as well as on the dehydrated samples of each with an incident neutron wavelength of $6 \AA$ corresponding to an energy resolution (FWHM) of $\Delta \mathrm{E} \sim 45 \mu \mathrm{eV}$. QENS experiments were performed in the temperature range $260-360 \mathrm{~K}$ using the hybrid time of flight spectrometer "FOCUS" at SINQ, Paul Scherrer Institut, Switzerland. The samples were placed in a flat rectangular aluminum can with an internal spacing of $0.2 \mathrm{~mm}$ (which ensures no more than $10 \%$ scattering) such that multiple scattering effects can be neglected. The quasielastic spectra were recorded in the $Q$ range of $0.4-1.6 \AA^{-1}$. The measured spectra were corrected for the detector efficiency with a standard vanadium sample and were normalized to the monitor intensity. As experimental Bragg peak positions are known from the diffraction pattern, during $Q$ binning such $Q$ values (where Bragg peaks occur) are avoided. The DAVE software ${ }^{25}$ developed by NIST was used to carry out the data reduction involving background subtraction, detector efficiency corrections, etc. Data from the dehydrated sample were used to estimate the contribution from the sample other than water. For dehydration, the sample was heated at $420 \mathrm{~K}$ under vacuum

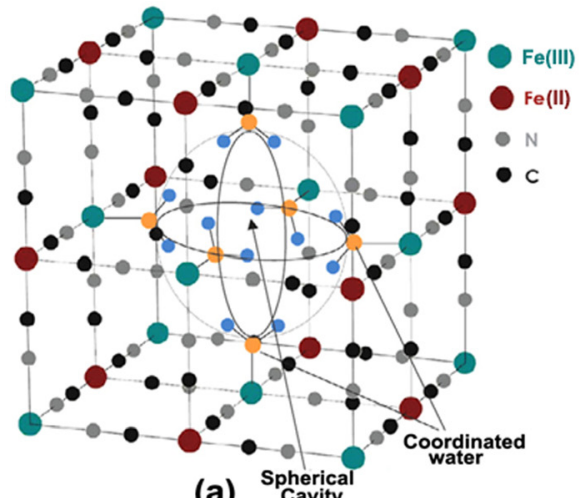

(a)

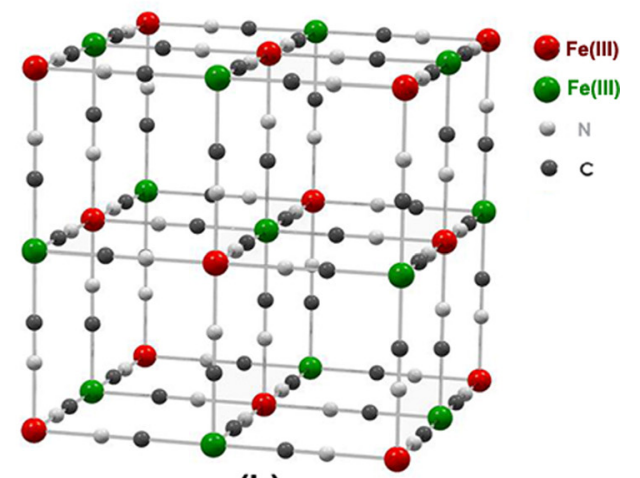

(b)
FIG. 1. Unit cell of (a) Prussian blue (PB), $\mathrm{Fe}(\mathrm{III})_{4}\left[\mathrm{Fe}(\mathrm{II})(\mathrm{CN})_{6}\right]_{3} \cdot 14 \mathrm{H}_{2} \mathrm{O}$ and (b) Prussian green (PG) or ferriferricyanide, $\quad\left(\mathrm{Fe}_{4}(\mathrm{III})\left[\mathrm{Fe}(\mathrm{III})(\mathrm{CN})_{6}\right]_{4} \cdot 16 \mathrm{H}_{2} \mathrm{O}\right)$. The spherical cavity formed by coordinated water molecules in PB is indicated. The non-coordinated water molecules are omitted for clarity. 
(15 mb) for a period of about $10 \mathrm{~h}$. The dehydrated sample was then sealed in a glove box and used for the neutron scattering measurements. The weight of the sample was recorded before and after dehydration process. The weight loss as observed after dehydration is also independently confirmed by the Thermo Gravimetric Analysis (TGA) measurements. Thermogravimetric measurements were carried out with Mettler thermogravimetric analyzer (TG 50). The thermograms are recorded in air atmosphere at a heating rate of $5^{\circ} \mathrm{C} \mathrm{min}-1$ in the temperature range $40-300^{\circ} \mathrm{C}$. The diffraction pattern recorded for the dehydrated sample confirms the same $f c c$ crystal structure with space group $F m 3 m$.

\section{RESULTS AND DISCUSSIONS}

In a neutron scattering experiment with a hydrogenous sample, the measured intensity is proportional to the double differential scattering cross section, which in turn is proportional to the incoherent scattering law $\mathrm{S}(\boldsymbol{Q}, \omega)$. Here $\boldsymbol{Q}$ is the wavevector transfer and $\omega$ is the angular frequency corresponding to the energy transfer, $\hbar \omega=E_{f}-E_{i}, E_{i}$ and $E_{f}$ being the initial and final energies, respectively, of the neutrons. In general, this scattering law can be written as ${ }^{11}$

$$
\mathrm{S}(Q, \omega)=A(Q) \delta(\omega)+[1-A(Q)] L(\Gamma, \omega),
$$

where the first term is the elastic part and the second is the quasielastic one. $L(\Gamma, \omega)$ is a Lorentzian function with a half width at half maxima (HWHM) $\Gamma$. The variation of HWHM, $\Gamma$ provides an information about the time scale of the motion. $\mathrm{A}(\mathrm{Q})$ is Elastic Incoherent Structure Factor (EISF). EISF is the space Fourier transform of the particle distribution, taken at infinite time and averaged over all the possible initial positions, providing information about the geometry of the molecular reorientations. It is convenient to analyse the data in terms of EISF, which is the fraction of the elastic intensity present in the total $\mathrm{S}(Q, \omega)$. It should be noted that EISF originates from the localized character of a motion, and the term $A(Q)$ is zero for an unrestricted translational diffusion process. However, under the effect of confinement, probability to find the particle at infinite time in the confined region will not be zero even for a translational diffusion process. Therefore, confinement gives rise to a finite elastic component in the scattering law.

Significant quasielastic (QE) broadening is observed for $\mathrm{PG}$ as well as PB over the instrument resolution whereas no
QE broadening is observed for the dehydrated samples. Therefore, the observed QE broadening corresponds to the dynamics of water molecules. This is also corroborated by the vibrational density of states (DOS) as obtained from the time of flight data for all the compounds and their dehydrated form using the following simplified expression: ${ }^{26}$

$$
g(E) \cong \frac{E}{Q^{2}}\left[1-\exp \left(-\frac{E}{K_{B} T}\right)\right] S(Q, E),
$$

where $\mathrm{K}_{\mathrm{B}}$ is Boltzman constant, $\mathrm{T}$ is temperature, and $\mathrm{E}$ is energy transfer. The peak at about $8 \mathrm{meV}$, corresponding to the intermolecular bending motions of the water molecules, ${ }^{20,26}$ observed in both PG and PB, however, is found to be absent in the dehydrated form as shown in Fig. 2. Absence of peak at $8 \mathrm{meV}$ in the dehydrated form suggests that sample is devoid of water molecules. The loss of water molecules in the PG and PB are clearly evident.

Thermogravimetric measurements have been carried out on PG and PB compounds, the observed thermograms are shown in Fig. 3. In PB, both coordinated and non-coordinated waters are weakly bound, and under heating, crystal water evolves at a lower temperature compared to that for PG. The thermogram of $P G$ is found to be very different than that of PB. It is found that PB shows a sharp weight loss compared to the $\mathrm{PG}$, indicating that the water molecules are relatively loosely bound in PB. The observed thermograms are also compared with thermogravimetric study for other PBA compounds $\mathrm{M}_{3}\left[\mathrm{Fe}(\mathrm{CN})_{6}\right]_{2} \cdot \mathrm{zH}_{2} \mathrm{O}(\mathrm{M}=\mathrm{Ni}, \mathrm{Zn}, \mathrm{Cd}, \mathrm{Mn}$, Co and $\mathrm{Cu}$ ) and found to be consistent. ${ }^{30} \mathrm{It}$ is clear that up to $200^{\circ} \mathrm{C}$ these compounds are stable and no decompositions take place.

Now we present the results of the QENS studies on both PG and PB compounds. QENS data as obtained from the dehydrated sample were subtracted from that of the corresponding hydrated samples (PG or PB) to extract the contribution from the water alone. Typical subtracted QENS spectra for PG and PB are shown in Fig. 4 at $Q=1.0 \AA^{-1}$ at $330 \mathrm{~K}$. It is evident that in case of PB more QE broadening is observed vis-à-vis $\mathrm{PG}$ indicating higher mobility of water molecules in $\mathrm{PB}$ compounds, which has spherical cavities due to vacant $\mathrm{Fe}(\mathrm{CN})_{6}$ sites. For both the compounds, QENS spectra are found to be comprised of at least two contributions, a narrow elastic peak that reflects scattering from species, which are static or moving slower than the instrumental resolution, and quasielastic broadening arising from the mobile species.
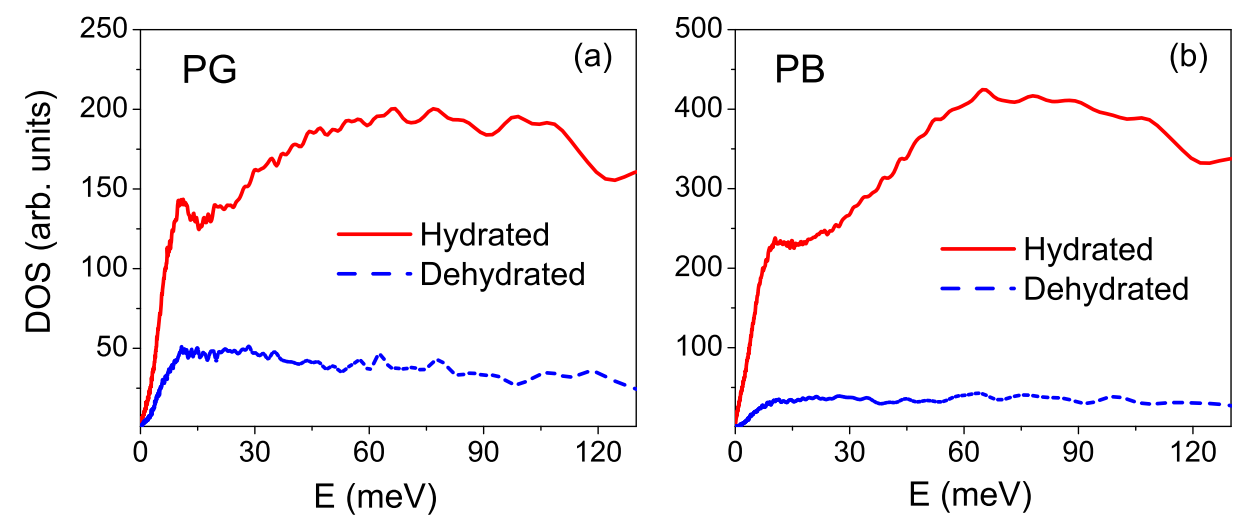

FIG. 2. Vibrational DOS in (a) PG and (b) $\mathrm{PB}$ in hydrated and dehydrated form at $300 \mathrm{~K}$. The peak $\sim 8 \mathrm{meV}$ corresponding to water molecules in PG and PB is not seen in the dehydrated samples. 


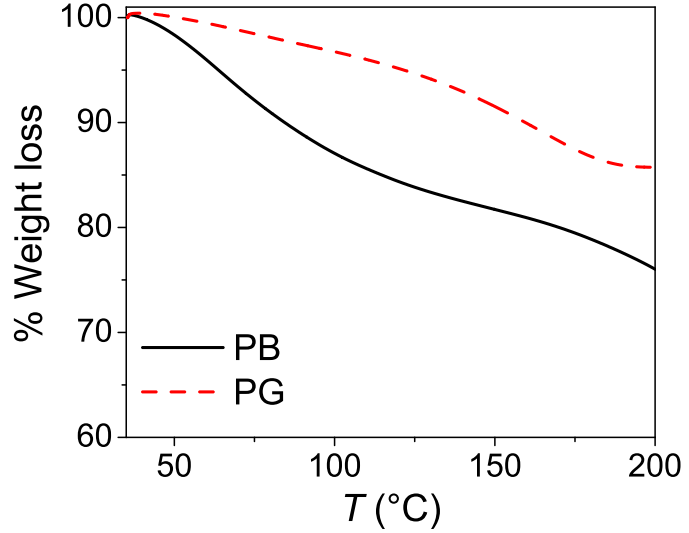

FIG. 3. The thermograms for $\mathrm{PB}\left(\mathrm{Fe}_{4}(\mathrm{III})\left[\mathrm{Fe}(\mathrm{II})(\mathrm{CN})_{6}\right]_{3} \cdot 14 \mathrm{H}_{2} \mathrm{O}\right)$ and $\mathrm{PG}$ $\left(\mathrm{Fe}_{4}(\mathrm{III})\left[\mathrm{Fe}(\mathrm{III})(\mathrm{CN})_{6}\right]_{4} \cdot 16 \mathrm{H}_{2} \mathrm{O}\right)$ compounds. Sharp loss in weight in $\mathrm{PB}$ compared to $\mathrm{PG}$ is evident.

On increase of temperature from $260 \mathrm{~K}$ to $360 \mathrm{~K}$, the QE broadening is found to increase in both the compounds. Typical evolutions in the QENS spectra for PG and $\mathrm{PB}$ at the observed temperature range $260-360 \mathrm{~K}$ are shown in the Figs. 5(a) and 5(b), respectively. It is found that the evolution in quasielastic broadening for PG (without any vacant $\mathrm{Fe}(\mathrm{CN})_{6}$ unit) is slower than $\mathrm{PB}$ (which have cavities due to vacant $\mathrm{Fe}(\mathrm{CN})_{6}$ units). To proceed with the analysis, it is required to separate out the elastic and quasielastic components of the subtracted data. For that, model scattering law as given in Eq. (1) is convoluted by the instrumental resolution function, and the parameters $A(Q)$ and $\Gamma(Q)$ were determined by least squares fit with the measured data. QENS spectra at different temperatures for both the compounds are satisfactorily described with Eq. (1). Typical data fits at different temperatures and $Q$ values are shown in Figs. 6 and 7 for PG and $\mathrm{PB}$ respectively. A finite EISF, $\mathrm{A}(\mathrm{Q})$ and a $Q$ dependent quasielastic broadening $(\Gamma(Q))$ are observed. Presence of an elastic contribution, $A(Q)$ in both PB and PG samples suggests that some water molecules are immobile within the time scale accessible by the spectrometer and/

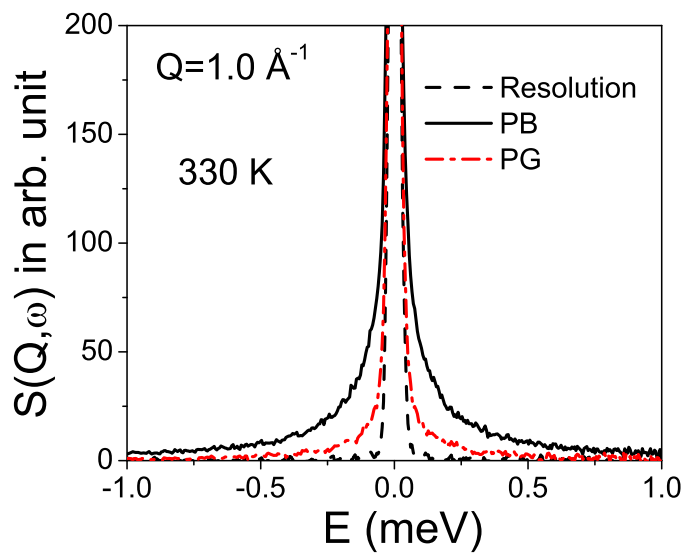

FIG. 4. Comparison of QENS spectra as obtained for $\mathrm{PG}$ and $\mathrm{PB}$ at $Q=1.0 \AA^{-1}$ at $330 \mathrm{~K}$. The instrument resolution as obtained from a standard vanadium sample is shown by a dashed line. Spectra have been normalized to maximum peak intensity of vanadium.
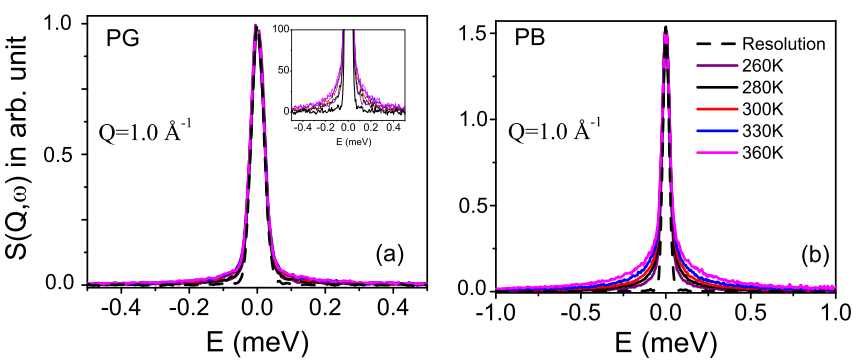

FIG. 5. QENS spectra recorded for (a) PG and (b) PB at different temperatures at $Q=1.0 \AA^{-1}$. Contribution from dehydrated sample has been subtracted. The dashed lines show the instrumental resolution. Spectra have been normalized to maximum peak intensity.

or are undergoing localised motion. The obtained EISF for both the compounds $\mathrm{PG}$ and $\mathrm{PB}$ are shown in Fig. 8. EISF based on the isotropic rotational model ${ }^{27}$ is also calculated and shown in Fig. 8 by a dashed line. It is clearly evident from the figure that for these compounds an isotropic rotation is also not a feasible model to describe the observed data. We will show that the localized translational diffusion model describes successfully the observed EISF and used in the present study.

The variation of obtained EISF at different temperatures for both the compounds indicates that there is an evolution of dynamics as the temperature is increased. However, this evolution is more pronounced in PB. The evolution in dynamics suggests that as temperature is increased, more number of water molecules contribute to the dynamics. Considering all the facts, a generalized scattering law for a system where fraction of the water molecules $\left(p_{x}\right)$ contribute to the dynamics can be written as

$$
\begin{aligned}
S(Q, \omega)= & \left(1-p_{x}\right) \delta(\omega) \\
& +p_{x}\left[A_{0}(Q) \delta(\omega)+\left(1-A_{0}(Q)\right) L(\Gamma, \omega)\right],
\end{aligned}
$$

where $\mathrm{A}_{0}(\mathrm{Q})$ is the model EISF and the total elastic fraction would be, $\left[p_{x} A_{0}(Q)+\left(1-p_{x}\right)\right]$. In case of translational diffusion of particles within a sphere of radius $a$ enclosed by impermeable boundary, $A_{0}(Q)$ can be given as $^{28}$

$$
A_{0}(Q)=\left[\frac{3 j_{1}(Q a)}{Q a}\right]^{2} .
$$

The effective EISF can be written as

$$
A(Q)=\left[p_{x}\left(\frac{3 j_{1}(Q a)}{Q a}\right)^{2}+\left(1-p_{x}\right)\right]
$$

Here, $j_{1}(Q a)$ is the first order spherical Bessel function. It is found that the above equation could describe the observed EISF for both the compounds, PB and PG very well at all the measured temperatures, as shown by the solid lines in Fig. 8. The fraction of the mobile water molecules, $p_{x}$, and radius of the spherical cavity within which the molecules undergo localized dynamics, $a$, are the parameters determined by least squares fitting of the 


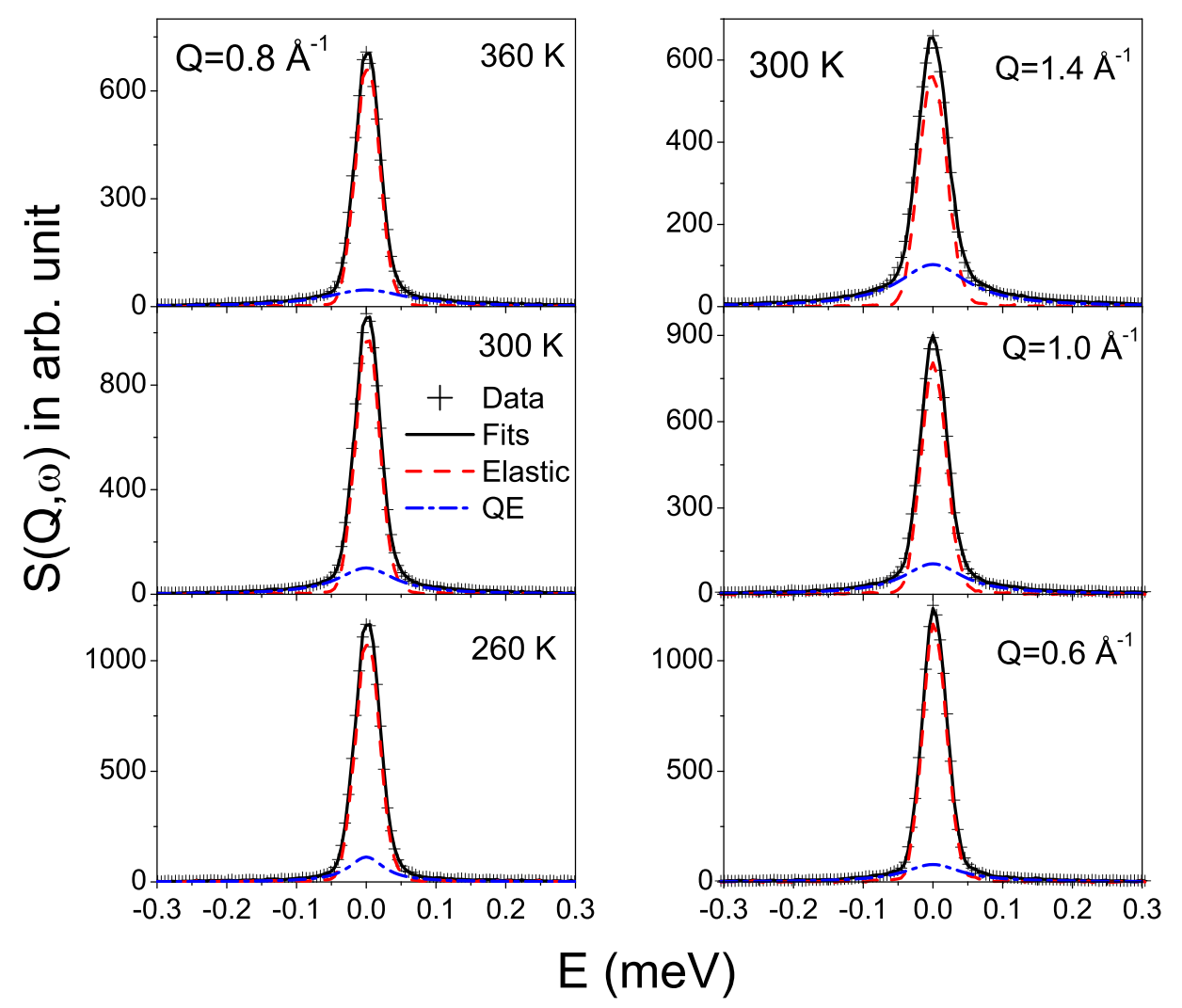

FIG. 6. Fitted subtracted QENS spectra for $\mathrm{PG}\left(\mathrm{Fe}_{4}(\mathrm{III})\left[\mathrm{Fe}(\mathrm{III})(\mathrm{CN})_{6}\right]_{4} \cdot 16 \mathrm{H}_{2} \mathrm{O}\right)$ (a) at different temperature at typical $\mathrm{Q}=0.8 \AA^{-1}$ and (b) at different $\mathrm{Q}$ values at $300 \mathrm{~K}$.

experimentally obtained EISF. For example at $260 \mathrm{~K}$, the values of $p_{x}$ and $a$ for water in $\mathrm{PG}$ are found to be 0.37 and $1.85 \AA$, respectively, suggesting that about $37 \%$ of water molecules which are mobile within a spherical volume having radius $1.85 \AA$ contribute to the observed dynamics. These results are found to be consistent with the available space for diffusion of water molecules in the structure of PG. ${ }^{6}$ However, for water in $\mathrm{PB}$ at $260 \mathrm{~K}$, the values of $p_{x}$ and $a$ are found to be 0.56 and $2.9 \AA$, respectively. It is clear that fraction of the water molecules $\left(p_{x}\right)$ contribute to the dynamics is more in case of $\mathrm{PB}$, compared to $\mathrm{PG}$. The derived value of the radius of the spherical

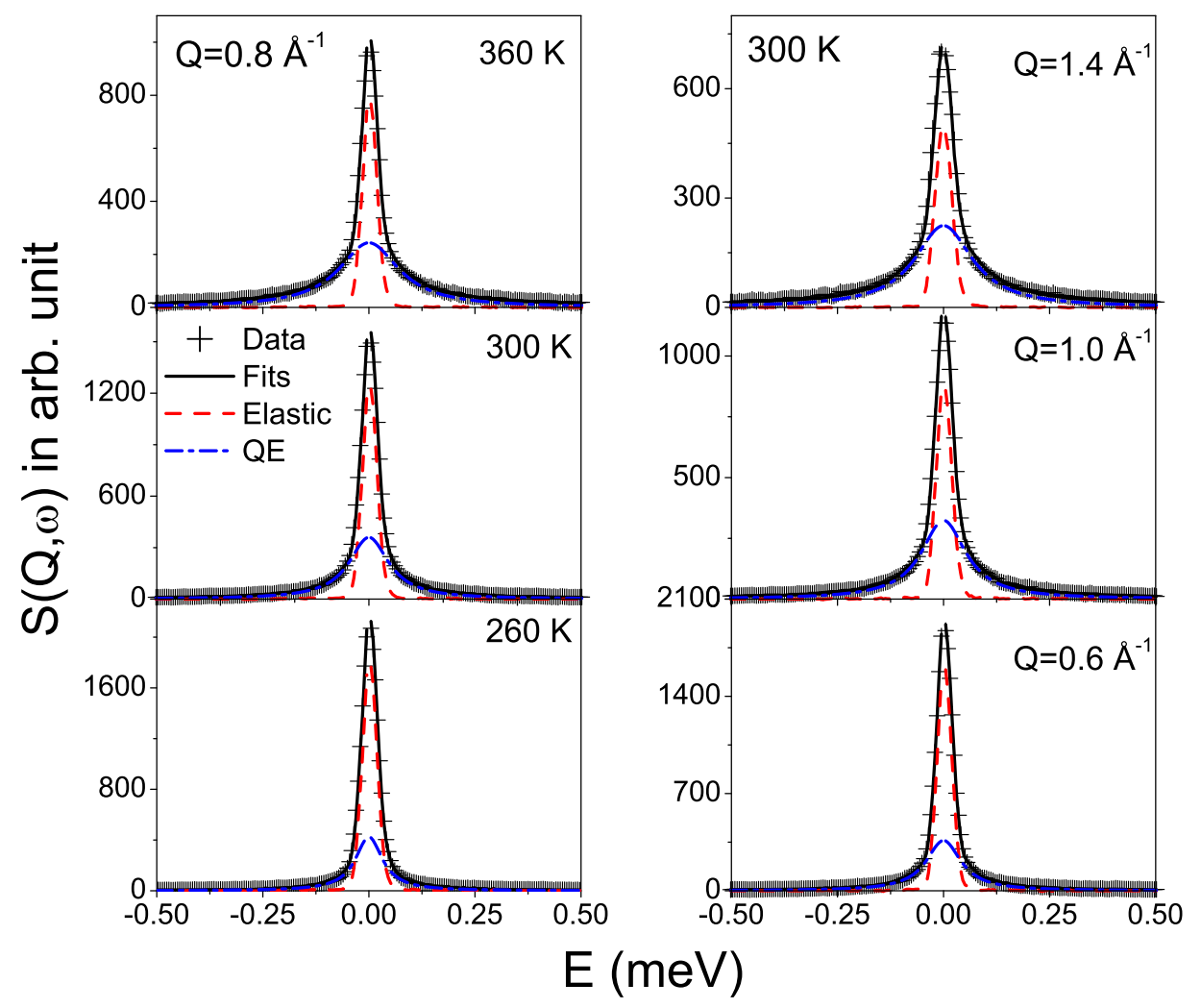

FIG. 7. Fitted subtracted QENS spectra for $\mathrm{PB}\left(\mathrm{Fe}_{4}(\mathrm{II})\left[\mathrm{Fe}(\mathrm{II})(\mathrm{CN})_{6}\right]_{3} .14 \mathrm{H}_{2} \mathrm{O}\right)$ (a) at different temperature at typical $\mathrm{Q}=0.8 \AA^{-1}$ and (b) at different $\mathrm{Q}$ values at $300 \mathrm{~K}$. 


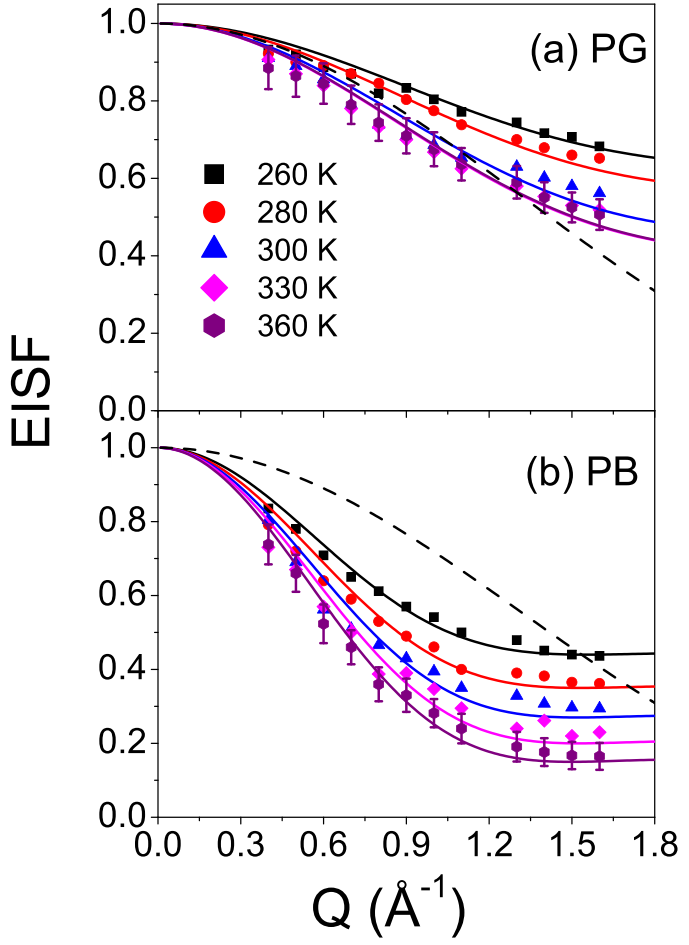

FIG. 8. Variation of EISF for (a) $\mathrm{PG}\left(\mathrm{Fe}_{4}(\mathrm{III})\left[\mathrm{Fe}(\mathrm{III})(\mathrm{CN})_{6}\right]_{4} \cdot 16 \mathrm{H}_{2} \mathrm{O}\right)$ and (b) $\mathrm{PB}\left(\mathrm{Fe}_{4}(\mathrm{III})\left[\mathrm{Fe}(\mathrm{II})(\mathrm{CN})_{6}\right]_{3} \cdot 14 \mathrm{H}_{2} \mathrm{O}\right)$ as obtained from QENS data with $Q$ at different temperatures. Solid lines show the fits using a localized translational diffusion model [Eq. (5)] with a fraction of immobile water molecules. The dashed lines represent the calculated EISF as per the model based on isotropic rotation.

volume (a) for PB is also found to be higher than that in PG and found to be very similar to the size of the cavity (radius $\sim 2.8 \AA$ ) formed in $\mathrm{PB}$ due to the vacant sites of $\mathrm{Fe}(\mathrm{CN})_{6} \cdot{ }^{9}$ Variation of $p_{\mathrm{x}}$ as well as diameter of spherical domain $(2 a)$ with temperature are shown in Figs. 9(a) and 9(b) for both the compounds. It is found that PB has most number of mobile water molecules. It is evident that fraction of mobile water molecules increases with increase in temperature. However, the size of spherical domain remains more or less constant at different temperatures. Value of $p_{\mathrm{x}}$ for $\mathrm{PB}$ and $\mathrm{PG}$ at $360 \mathrm{~K}$ are found to be 0.85 and 0.59 , respectively.

The variations of the HWHM of QE component $(\Gamma)$ as a function of $Q$ for PB and PG at different temperatures are shown in Fig. 10. It is very clear that the variation of the QE width is very different than that observed in bulk water. ${ }^{29} \mathrm{In}$ case of bulk water, ${ }^{29}$ HWHM increases linearly with $Q^{2}$ and then saturates to a constant value indicating a dynamics typified by jump diffusion. Here, particularly at low Q, the HWHM shows significantly different behavior. At lower $Q$ $(Q a<\pi)$, i.e., when larger distances are probed, behavior of HWHM approaches a constant value, which is independent of $Q$. This is due to the fact that in this length scale, the water molecules are seen to perform localised dynamics. Therefore, behaviour of quasielastic width in this $Q$ range resembles that of rotational motion. However, at larger $Q$ values $(Q a>\pi)$, where small distances are probed, usual $\mathrm{D} Q^{2}$ behaviour, corresponding to a translational motion in an infinite medium, is recovered since in this length scale, i.e.,

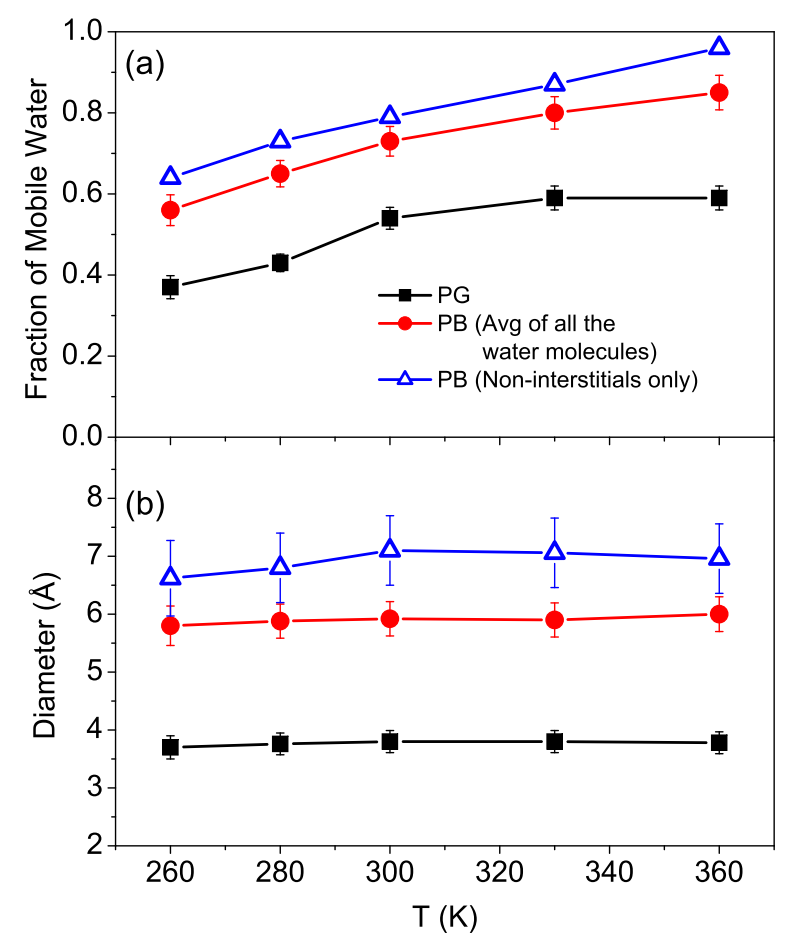

FIG. 9. Variation of (a) fraction of water molecules taking part in the dynamics and (b) diameter of confining volume in $\mathrm{PB}\left(\mathrm{Fe}_{4}(\mathrm{III})\left[\mathrm{Fe}(\mathrm{III})(\mathrm{CN})_{6}\right]_{3} \cdot 14 \mathrm{H}_{2} \mathrm{O}\right)$ and $\mathrm{PG}\left(\mathrm{Fe}_{4}(\mathrm{III})\left[\mathrm{Fe}(\mathrm{III})(\mathrm{CN})_{6}\right]_{4} \cdot 16 \mathrm{H}_{2} \mathrm{O}\right)$. Open symbols $(\Delta)$ correspond to the fraction of mobile water molecules exist only at the non-interstitial sites in PB (see the text).

when probed inside the cavity, confined wall boundaries are not seen.

The model scattering function for the water molecules performing localised translational diffusion inside a spherical cavity can be given as ${ }^{28}$

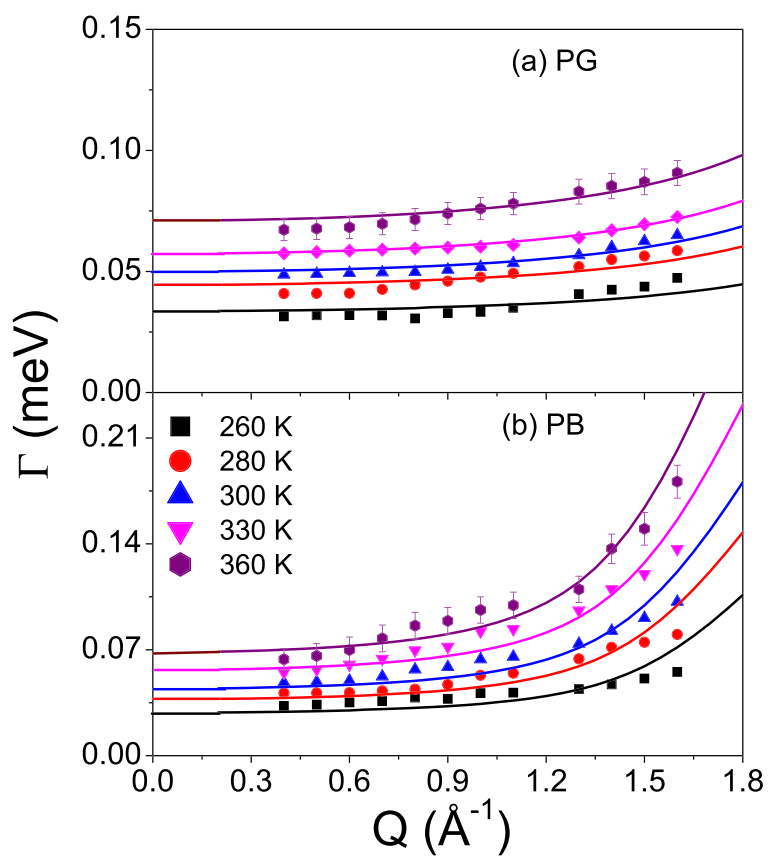

FIG. 10. Variation of HWHM of a Lorentzian representing water dynamics in (a) $\mathrm{PG} \quad\left(\mathrm{Fe}_{4}(\mathrm{III})\left[\mathrm{Fe}(\mathrm{III})(\mathrm{CN})_{6}\right]_{4} \cdot 16 \mathrm{H}_{2} \mathrm{O}\right)$ and (b) $\mathrm{PB} \quad\left(\mathrm{Fe}_{4}(\mathrm{III})[\mathrm{Fe}\right.$ (II) $\left.(\mathrm{CN})_{6}\right]_{3} \cdot 14 \mathrm{H}_{2} \mathrm{O}$ ) with $Q$. Solid lines are the fit with a model based on localized translational diffusion as described in Eq. (6). 


$$
S(Q, \omega)=\left(1-p_{x}\right) \delta(\omega)+p_{x}\left[A_{0}^{0}(Q a) \delta(\omega)+\frac{1}{\pi} \sum_{\{l, n\} \neq\{0,0\}}(2 l+1) A_{n}^{l}(Q a) \frac{\left(x_{n}^{l}\right)^{2} D / a^{2}}{\left[\left(x_{n}^{l}\right)^{2} D / a^{2}\right]^{2}+\omega^{2}}\right] .
$$

Here, the first term represents the elastic contribution due to immobile water molecules. The second term represents the scattering function for localised translational diffusion weighted by the fraction of mobile water molecules. Here, $D$ is the diffusion coefficient and $x_{n}^{l}=a \sqrt{\lambda_{n}^{l}}$ are dimensionless numbers, whose values of first 99 are tabulated in Ref. 28. $A_{n}^{l}(Q a)$ for different $n$ and $l$ can be calculated by using the expression given in Ref. 28. In the limit $Q a \rightarrow \infty$, the second term of Eq. (6) reduces to a scattering law for translational motion (a single Lorentzian) in infinite medium.

Since no analytical expression exists for the HWHM of the quasielastic part unlike that in case of EISF, the HWHM can be calculated numerically (using Eq. (6)) for given values of $a$ and $D$. The least-squares fitting method is used to describe the observed QE width with $D$ as parameter, while the values of $a$ are already known from the fit of the EISF. Figs. 10(a) and 10(b) show the fit of the QE widths as obtained assuming the localised translational diffusion model to describe the dynamics of water in the PB and PG respectively. At $260 \mathrm{~K}$, diffusion coefficient, for water in PG is found to be $0.41 \times 10^{-5} \mathrm{~cm}^{2} / \mathrm{s}$ which is half of the observed value in $\mathrm{PB}\left(\sim 0.84 \times 10^{-5} \mathrm{~cm}^{2} / \mathrm{s}\right)$. Slower dynamics of water molecules in PG could be understood as water molecules in PG are only at interstitial position and are more geometrical constrained compared to $\mathrm{PB}$ which has more empty space due to vacant $\mathrm{Fe}(\mathrm{CN})_{6}$ units. At room temperature $(300 \mathrm{~K})$, diffusion constant for water in PG and PB are found to be 0.63 and $1.36 \times 10^{-5} \mathrm{~cm}^{2} / \mathrm{s}$ which is much hindered compared to bulk water $\left(\sim 2.5 \times 10^{-5} \mathrm{~cm}^{2} / \mathrm{s}\right)$. Diffusion coefficient of water is found to increase with temperature in both PG and PB compounds and at $360 \mathrm{~K}$, the values of diffusion constant are found to be 0.89 and $2.15\left(\times 10^{-5} \mathrm{~cm}^{2} / \mathrm{s}\right)$. Variations of diffusion coefficient of water in both PB and PG with the temperature are shown in Fig. 11. It is evident that the diffusion coefficients follow the Arrhenius behaviour for all the compounds and activation energies for water are found to be 1.34 and $1.71 \mathrm{Kcal} / \mathrm{mol}$ for $\mathrm{PG}$ and $\mathrm{PB}$, respectively.

From the present study, it is clear that dynamics of water molecules in PG is more hindered compared to PB. This could be understood from the fact that in PG there is no cavity which means that not much free space available for water molecules to diffuse. Diffusion in PG is hindered mainly due to the geometrical constraint. It may be noted that observed dynamical behavior in PB is actually averaged over of all three kinds of water molecules existing in the system. It may be fair to consider that the water molecules present in the interstitial sites have similar dynamical behaviour in both $\mathrm{PB}$ and PG, and with that assumption, it is possible to extract the dynamical information of other water molecules which are not in the interstitial sites (i.e., coordinated as well as noncoordinated water molecules present in the spherical cavity formed by vacant $\mathrm{Fe}(\mathrm{CN})_{6}$ units) in $\mathrm{PB}$. In that case, the observed EISF in PB can be written as

$$
\begin{aligned}
A(Q)= & \alpha_{1}\left[p_{I}\left(\frac{3 j_{1}\left(Q a_{I}\right)}{Q a_{I}}\right)^{2}+\left(1-p_{I}\right)\right] \\
& +\left(1-\alpha_{1}\right)\left[p_{C}\left(\frac{3 j_{1}\left(Q a_{C}\right)}{Q a_{C}}\right)^{2}+\left(1-p_{C}\right)\right] .
\end{aligned}
$$

The first term corresponds to the water molecules at the interstitial sites, where $\alpha_{1}$ is the fraction of total noncoordinated water molecules at interstitial sites out of the total number of water molecules, and $\mathrm{p}_{I}$ is the fraction that contributes to the dynamics, $a_{\mathrm{I}}$ is the radius of spherical volume in which they undergo localised diffusion. $p_{\mathrm{c}}$ corresponds to the fraction of mobile water molecules at and around spherical cavity created due to vacant $\mathrm{Fe}(\mathrm{CN})_{6}$ units, and $a_{\mathrm{c}}$ is the size of domain in which these water molecules diffuse. As it is known from the structural study, that in PB, out of 14 water molecules, 4 are at the interstitial position and 10 are at the spherical cavity created due to vacant $\mathrm{Fe}$ $(\mathrm{CN})_{6}$ sites out of which 6 are coordinated and 4 are noncoordinated. Therefore, $\alpha_{1}$ can be fixed to $4 / 14=0.286$. Since we have assumed that the water molecules in the interstitial site in PB and PG are dynamically similar, $a_{I}$ and $p_{I}$ are fixed to the values, obtained from the PG system. The parameters correspond to the non-interstitial water molecules are $a_{\mathrm{c}}$ and $p_{\mathrm{c}}$, are obtained using Eq. (7) by a least squares fit of the observed EISF. Quality of fit is improved significantly as shown in Fig. 12. The derived values of $a_{\mathrm{c}}$ and $p_{\mathrm{c}}$ are also shown in Fig. 9 by open $(\Delta)$ symbols. While at $260 \mathrm{~K}$ about $56 \%$ of water molecules in the spherical cavity were found to be mobile, the same is now found to be $64 \%$. Similarly at

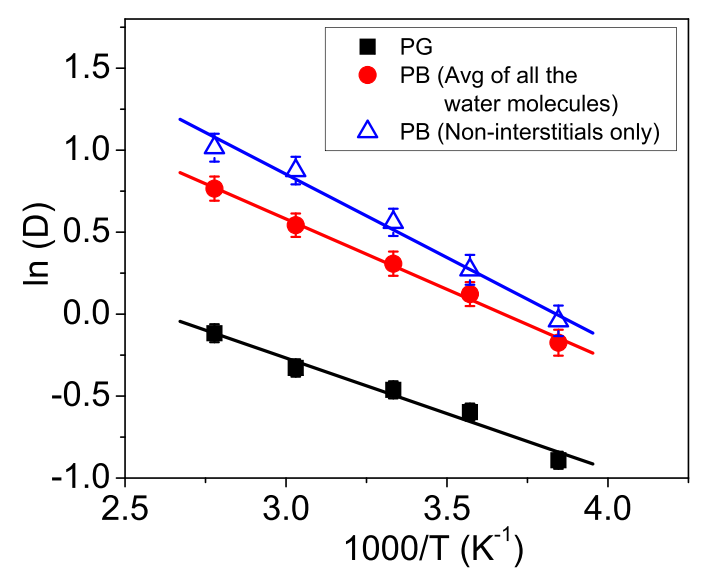

FIG. 11. Variation of diffusion constant (D) for PB and PG in the temperature range $260-360 \mathrm{~K}$. Open symbols $(\Delta)$ correspond to the fraction of mobile water molecules exist only at the non-interstitial sites in PB (see the text). 


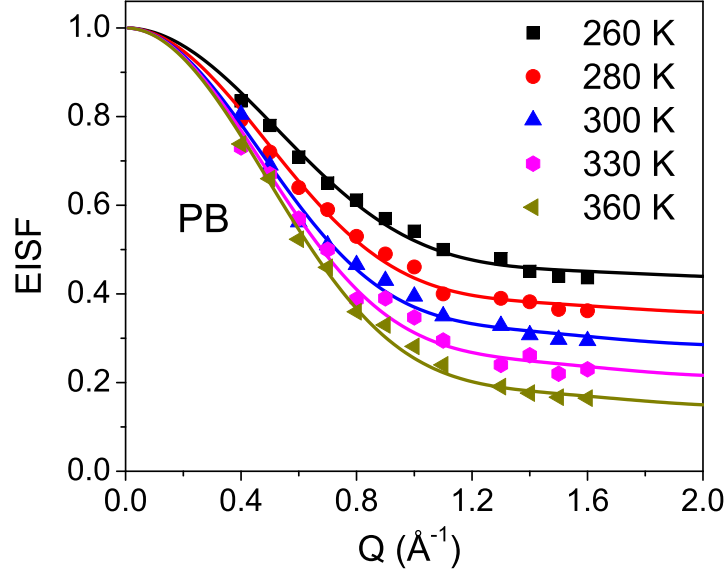

FIG. 12. Fit of observed EISF with Eq. 7 with the assumption that the water molecules in the interstitial sites in both PB and PG systems have similar dynamical characteristics (similar $a_{\mathrm{I}}$ and $\mathrm{p}_{I}$ as described in Eq. (7)).

$360 \mathrm{~K}$ almost all the water molecules at the spherical cavity $(\sim 96 \%)$ are found to undergo localized translational diffusion compared to $85 \%$ found earlier. Similar methodology was used to calculate the diffusion constant, $D$, of noninterstitial water molecules only for PB and the obtained values are shown in Fig. 11 by open $(\Delta)$ symbols. It may be noted that these values are slightly more than that the average ones. This is consistent with the fact that the values obtained earlier were average of the two kinds of water in which one was lower than the other and so the average was lower. This shows that we could estimate the dynamics of water molecules separately for the different sites and the analysis is consistent.

\section{CONCLUSION}

Dynamics of water in hexacyanometallates family $\left(\mathrm{A}_{\mathrm{x}}\left[\mathrm{B}(\mathrm{CN})_{6}\right] \mathrm{y} . \mathrm{zH}_{2} \mathrm{O}\right)$ i.e., Prussian blue $(\mathrm{x} / \mathrm{y}>1)$, $\mathrm{Fe}(\mathrm{III})_{4}\left[\mathrm{Fe}(\mathrm{II})(\mathrm{CN})_{6}\right]_{3} \cdot 14 \mathrm{H}_{2} \mathrm{O}$ and Prussian green $(\mathrm{x} / \mathrm{y}=1)$, $\mathrm{Fe}(\mathrm{III})_{4}\left[\mathrm{Fe}(\mathrm{III})(\mathrm{CN})_{6}\right]_{4} \cdot 16 \mathrm{H}_{2} \mathrm{O}$ have been studied using quasielastic neutron scattering technique in the temperature range $260-360 \mathrm{~K}$. Combining the results from these two compounds, dynamical behaviour of different kinds of water molecules present in these compounds has been elucidated. In $\mathrm{PB}$, there exists spherical cavities created due to the vacant sites of $\mathrm{Fe}(\mathrm{CN})_{6}$ units and there are three structurally distinguished water molecules: (i) coordinated water molecules at empty nitrogen sites, (ii) non-coordinated water molecules in the spherical cavities, and (iii) at interstitial sites. Whereas PG does not have any vacant units and only noncoordinated water molecule exist at interstitial sites.

Neutron density of states showed that the dehydrated samples are devoid of water and therefore contribution of the water alone could be obtained by subtracting the data of the dehydrated sample. Presence of much larger quasielastic broadening in $\mathrm{PB}$ compared to $\mathrm{PG}$, clearly indicates that water molecules diffuse faster in PB. Also the evolution of the dynamics with temperature is found to be much more pronounced in PB than PG. Detailed data analysis showed that the water molecules are localized and undergo translational diffusion within a confined domain. This is found to be the case for both PB and PG systems. The average diffusion coefficient associated with the crystal water is found to be much larger in PB compared to PG. The domain within which the water molecules are mobile is also found to be larger in PB. This is consistent with the geometry of the structure of the two systems. The domain of dynamics in PB is large due to the existence of spherical cavity ( $\sim 6 \mathrm{~A}$ dia) within which the water can diffuse whereas in PG no such cavity exists and so the domain of dynamics could be much smaller. A better estimate of the dynamical parameters, for the water molecules that exist in the cavities, has been obtained by assuming that the water molecules at the interstitial sites in both the systems have similar dynamical characters.

In summary, a consistent picture of the dynamical behavior of the water molecules in the Prussian blue analogues has been obtained by combining the data of the PB and PG systems having different geometrical conformations.

${ }^{1}$ O. Kahn, Molecular Magnetism (VCH, New York, 1993).

${ }^{2}$ J. S. Miller and A. J. Epstein, Angew. Chem., Int. Ed. Engl. 33, 385 (1994).

${ }^{3}$ S. Ferlay, T. Mallah, R. Ouahes, P. Veillet, and M. Verdaguer, Nature (London) 378, 701 (1995).

${ }^{4}$ O. Sato, T. Iyoda, A. Fujishima, and K. Hashimoto, Science 271, 49 (1996).

${ }^{5}$ S. I. Ohkoshi, K. I. Arai, Y. Sato, and K. Hashimoto, Nature Mater. 3,857 (2004).

${ }^{6}$ A. Kumar, S. M. Yusuf, and L. Keller, Phys. Rev. B 71, 054414 (2005).

${ }^{7}$ A. Kumar, S. M. Yusuf, L. Keller, and J. V. Yakhmi, Phys. Rev. Lett. 101, 207206 (2008).

${ }^{8}$ S. M. Yusuf, A. Kumar, and J. V. Yakhmi, Applied Phys. Lett. 95, 182506 (2009).

${ }^{9}$ P. Herren, P. Fischer, A. Ludi, and W. Halg, Inorg. Chem. 19, 956 (1980).

${ }^{10}$ V. K. Sharma, S. Mitra, A. Kumar, S. M. Yusuf, F. Juranyi, and R. Mukhopadhyay, J. Phys. Condensed Mater. 23, 446002 (2011).

${ }^{11}$ M. Bée, Quasielastic Neutron Scattering (Adam Hilger, Bristol, 1988).

${ }^{12}$ D. R. Cole, E. Mamontov, and G. Rother, in Neutron Applications in Earth, Energy and Environmental Sciences, edited by L. Liang, R. Rinaldi, and H. Schober (Springer-Verlag, Berlin, 2009), Chapter 19.

${ }^{13}$ C. Dore, F. Coveney, and M.-C. Bellissent-Funel, in Recent Developments in the Physics of Fluids, edited by W. S. Howells and K. Soper (Adam Hilger, 1992), p. 299.

${ }^{14}$ S. Mitra, S. A. Prabhudesai, D. Chakraborty, V. K. Sharma, M. Vicente, J. P. Embs, and R. Mukhopadhyay, Phys. Rev. E 87, 062317 (2013).

${ }^{15}$ R. R. Dessai, V. K. Sharma, S. A. Prabhudesai, S. Mitra, J. A. E. Desa, and R. Mukhopadhyay, J. Phys. Soc. Jpn. 82, SA008 (2013).

${ }^{16}$ V. K. Sharma, S. Mitra, P. Singh, F. Jura'ny, and R. Mukhopadhyay, Eur. Phys. J. ST 189, 217 (2010).

${ }^{17}$ V. K. Sharma, P. S. Singh, S. Gautam, S. Mitra, and R. Mukhopadhyay, Chem. Phys. Lett. 478, 56 (2009).

${ }^{18}$ V. K. Sharma, P. S. Singh, S. Gautam, P. Maheshwari, D. Dutta, and R. Mukhopadhyay, J. Membr. Sci. 326, 667 (2009).

${ }^{19}$ S. Mitra, R. Mukhopadhyay, I. Tsukushi, and S. Ikeda, J. Phys.: Condensed Mater. 13, 8455 (2001).

${ }^{20}$ M.-C. Bellissent-Funel, S. H. Chen, and J.-M. Zanotti, Phys. Rev. E 51, 4558 (1995).

${ }^{21}$ V. Garcia Sakai, E. Mamontov, J. W. Lynn, L. Viciu, and R. J. Cava, Phys. Rev. B 75, 014505 (2007).

${ }^{22}$ J. Teixeira, J.-M. Zanotti, M.-C. Bellissent-Funel, and S. H. Chen, Physica B 234-236, 370 (1997).

${ }^{23}$ T. Tsukahara, W. Mizutani, K. Mawatari, and T. Kitamori, J. Phys. Chem. B 113, 10808 (2009).

${ }^{24}$ P. Demontis, J. G. Jonzalez, H. Jobic, and G. B. Suffritti, J. Phys. Chem. C 114, 18612 (2010). 
${ }^{25}$ R. T. Azuah, L. R. Kneller, Y. Qiu, P. L. W. Tregenna-Piggott, C. M. Brown, J. R. D. Copley, and R. M. Dimeo, J. Res. Natl. Inst. Stan. Technol. 114, 341 (2009).

${ }^{26}$ D. Russo, J. Teixeira, L. Kneller, J. R. D. Copley, J. Ollivier, S. Perticaroli, E. Pellegrini, and M. A. Ganzalez, J. Am. Chem. Soc. 133, 4882 (2011).
${ }^{27}$ V. F. Sears, Can. J. Phys. 44, 1299 (1966).

${ }^{28}$ F. Volino and A. J. Dianoux, Mol. Phys. 41, 271 (1980).

${ }^{29}$ J. Teixeira, M.-C. Bellissent-Funel, S. H. Chen, and A. J. Dianoux, Phys. Rev. A 31, 1913 (1985).

${ }^{30}$ R. Martınez-Garcia, M. Knobel, and E. Reguera, J. Phys. Chem. B 110, 7296 (2006). 